\title{
Prostaglandin E2 is a key factor for CCR7 surface expression and migration of monocyte-derived dendritic cells
}

Elke Scandella, Ying Men, Silke Gillessen, Reinhold Förster, and Marcus Groettrup

Dendritic cells (DCs) are potent antigenpresenting cells that are able to initiate and modulate immune responses and are hence exploited as cellular vaccines for immunotherapy. Their capacity to migrate from peripheral tissues to the T-cell areas of draining lymph nodes is crucial for the priming of T lymphocytes. In this study, we investigated how the maturation of human monocyte-derived DCs (MoDCs) by several different stimuli under serum-free conditions affected their T-cell stimulatory function, cytokine secretion, and migratory behavior. Surprisingly, we found that for all maturation stimuli tested, the addition of prosta-

\section{Introduction}

Dendritic cells (DCs) are professional antigen-presenting cells that are uniquely able to stimulate naive $\mathrm{T}$ cells and initiate and control immune responses ${ }^{1}$ through the activation of T-helper $\left(\mathrm{T}_{\mathrm{H}}\right)$ cells of the $T_{H} 1, T_{H} 2$, or $T_{H}$ regulatory phenotype. ${ }^{2-4}$ They reside in an immature stage in peripheral tissues, where they capture antigens such as invading bacteria, viruses, or damaged tissue. Upon encountering antigens, but also in response to proinflammatory cytokines or T-cell-derived signals such as CD40 ligand (CD40L), DCs lose their phagocytic capacity and up-regulate costimulatory proteins, as well as major histocompatibility complex class I and II molecules presenting processed antigen..$^{5-7}$ At the same time, DCs secrete large amounts of inflammatory cytokines, such as tumor necrosis factor- $\alpha$ (TNF- $\alpha)$, interleukin- $1 \beta$ (IL-1 $\beta$ ), and IL-6, as well as chemokines (eg, CCL3 (macrophage inflammatory pro-

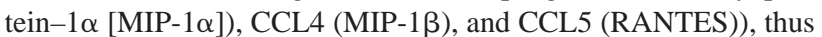
promoting the recruitment of monocytes and immature DCs into inflamed tissues. ${ }^{8}$ Most importantly, DCs acquire the ability to migrate from peripheral tissues to the T-cell areas of draining lymphoid organs; this ability is pivotal for their capacity to initiate an immune response and is tightly regulated by several mechanisms. Migrating leukocytes express adhesion molecules, such as selectins and integrins, for the interaction with endothelial cells, along with chemokine receptors, which are the sensors for chemotactic gradients. ${ }^{9}$ Various receptors for inflammatory chemokines, including CCR1, CCR2, CCR5, and CXCR1, are expressed on immature DCs, which therefore chemotactically respond to their cognate ligands CCL3, CCL2 (monocyte chemoattractant protein1), CCL4, and CXCL8 (IL-8), respectively. 8,10 During maturation, DCs lose their responsiveness to most of these inflammatory chemokines through receptor down-regulation or desensitization. Simultaneously, DCs up-regulate chemokine receptors for constitutively expressed chemokines, such as CXCR4 or CCR7, and acquire responsiveness to CCL21 (secondary lymphoid tissue chemokine [SLC]/6Ckine) and CCL19 (EBI1 ligand chemokine/ MIP-3ß), which are expressed in lymphatic vessels and the T-cell areas of lymph nodes. ${ }^{11,12}$ Expression of functional CCR7 is particularly important, as DCs from mice lacking CCR7 fail to migrate into draining lymph nodes. ${ }^{13}$ In addition, lipid mediators, such as the cysteinyl leukotriene C4, act on DCs through an autocrine mechanism, turning on chemotaxis in response to CCL19, and the interaction of CD40 with CD40L regulates migration of DCs from skin to draining lymph nodes. ${ }^{14-16}$

These unique features of DCs are increasingly exploited for the design of DC-based vaccines in immunotherapy since sufficient numbers of monocyte-derived DCs (MoDCs) can be obtained through in vitro differentiation of monocytes in the presence of granuloctye-macrophage colony-stimulating factor (GM-CSF) and IL-4. ${ }^{17,18}$ Unfortunately, little is known about migratory capacities of MoDCs generated under serum-free conditions for use as cellular vaccines in therapeutic application. It has been shown that 
immature MoDCs fail to migrate to lymph nodes, ${ }^{19}$ most likely owing to a lack of CCR7 expression, and consequently prime $\mathrm{T}_{\mathrm{H}}$ regulatory cells. ${ }^{20,21}$ Therefore, the use of matured MoDCs is crucial for the initiation of cytotoxic immune responses. Currently, several groups use a cocktail of proinflammatory mediators, such as TNF- $\alpha$, IL-1 $\beta$, IL-6, and prostaglandin E2 (PGE2), to mature MoDCs for application in immunotherapy.22-24 The lipid molecule PGE2 especially is a potent modulator of immune responses ${ }^{25}$ and has been reported to enhance T-cell stimulatory capacity and mean migratory activity of MoDCs ${ }^{24}$ generated under serum-free conditions. Furthermore, PGE2 supports the maturation of MoDCs stimulated with TNF- $\alpha .^{26,27}$ Concurrently, it has been shown that PGE2 down-regulates the production of the $\mathrm{T}_{\mathrm{H}} 1$-inducing cytokine IL-12p70 and enhances the production of IL-10, thus leading to a shift of the T-helper cell response toward the $\mathrm{T}_{\mathrm{H}} 2$-type. ${ }^{28} \mathrm{It}$ is still a matter of debate as to whether a $\mathrm{T}_{\mathrm{H}} 2$ bias through PGE2 negatively affects an antitumor response because, although $\mathrm{T}_{\mathrm{H}} 1$ differentiation is critical for an effective immune defense against some tumors, ${ }^{29,30}$ the contribution of the $\mathrm{T}_{\mathrm{H}} 2$ cytokine IL- 4 can be important for tumor eradication as well. ${ }^{31}$

PGE2 acts through $4 \mathrm{G}$ protein-coupled receptors, designated EP1, EP2, EP3, and EP4, that display different tissue distribution and deliver distinct intracellular signals. ${ }^{32,33}$ The $2 \mathrm{G}_{\mathrm{s}}$ proteincoupled receptors EP2 and EP4, which both mediate their signal through elevated cyclic adenosine monophosphate (cAMP), are expressed on monocytoid cells, ${ }^{34,35}$ but nothing is yet known about prostaglandin receptors expressed on DCs.

In this study, we have examined the effect of PGE2 on phenotypical and functional maturation of MoDCs generated under serum-free conditions with the use of 3 different stimuli. In particular, we analyzed surface expression of the chemokine receptors CCR7, CCR1, CCR5, and CXCR4 on immature and mature MoDCs, as well as their migratory capacity in response to CCL19 and CCL21. Our experiments identified PGE2 as a proinflammatory agent that generally enhances T-cell stimulatory capacity of MoDCs and, most importantly, not only supports CCR7 surface expression of MoDCs during maturation but is also able to strongly promote migration toward lymph node-derived chemokines.

\section{Materials and methods}

\section{Generation of MoDCs}

MoDCs were generated from human peripheral blood mononuclear cells (PBMCs) as previously described.$^{17}$ In brief, PBMCs were separated by standard density gradient centrifugation on Ficoll-Paque (Pharmacia, Uppsala, Sweden), resuspended at $4 \times 10^{6}$ cells per milliliter in AIM V medium (Invitrogen, Groningen, The Netherlands), and allowed to adhere to plastic for 1 hour at $37^{\circ} \mathrm{C}$. Nonadherent cells were removed, and the remaining cells were cultured in AIM $\mathrm{V}$ medium containing $50 \mathrm{ng} / \mathrm{mL}$ GM-CSF (Leukomax) (Novartis, Basel, Switzerland) and 1000 U/mL IL-4 (Strathmann, Hamburg, Germany). Alternatively, monocytes were purified by positive selection with anti-CD14-conjugated magnetic microbeads (Miltenyi, Bergisch Gladbach, Germany) and cultivated at $1 \times 10^{6}$ cells per milliliter in the same medium. On day 6 , nonadherent and loosely adherent cells were harvested and recultured $\left(5 \times 10^{5}\right.$ cells per milliliter) in cytokine-containing medium in the absence or presence of either a cocktail of proinflammatory cytokines ( $20 \mathrm{ng} / \mathrm{mL}$ TNF- $\alpha, 10 \mathrm{ng} / \mathrm{mL} \mathrm{IL}-1 \beta$, and $1000 \mathrm{U} / \mathrm{mL}$ IL-6, all purchased from Strathmann), 500 ng sCD40L (Immunex, Seattle, WA), or $20 \mu \mathrm{g} / \mathrm{mL}$ polyI:C (Sigma, Buchs, Switzerland) to induce maturation for an additional 48 hours. Where indicated, $1 \mu \mathrm{g} / \mathrm{mL}$ PGE2 (Prostin E2) (Pharmacia and Upjohn, Dübendorf, Switzerland), $1 \mu \mathrm{M}$ forskolin, (Sigma), $10 \mu \mathrm{g} / \mathrm{mL}$ cholera toxin (Alexis Biochemicals, Läufelfingen, Switzerland), $50 \mathrm{ng} / \mathrm{mL}$ pertussis toxin (Alexis Biochemicals), or $1 \mu \mathrm{M} 11-$ deoxy-PGE1 (Alexis Biochemicals) were added simultaneously.

\section{T-cell purification and MoDC cocultures}

Naive $\mathrm{CD} 4{ }^{+} \mathrm{CD} 45 \mathrm{RA}^{+} \mathrm{T}$ cells were purified from monocyte-depleted PBMCs by negative selection by means of the $\mathrm{CD}^{+} / \mathrm{CD} 45 \mathrm{RO}^{-}$Multi Sort Kit (Miltenyi). This method yielded purified (exceeding 98\%) $\mathrm{CD}^{+} / \mathrm{CD} 45 \mathrm{RA}^{+} /$ $\mathrm{CD} 4 \mathrm{RO}^{-} \mathrm{T}$ cells as assessed by flow cytometry (data not shown). For mixed lymphocyte reaction experiments, mature and immature allogeneic MoDCs were extensively washed, irradiated (30 Gy), and cultured at different numbers in duplicate with $1 \times 10^{5} \mathrm{CD}^{+}{ }^{+} \mathrm{CD} 45 \mathrm{RA}{ }^{+} \mathrm{T}$ cells in 96-well flat-bottom plates. On day 4 of coculture, ${ }^{3} \mathrm{H}$-thymidine $(1 \mu \mathrm{Ci}$ per well [ $37 \mu \mathrm{Bq}$ per well]) was added, and incorporation was tested after 14 to 16 hours. For T-cell-priming experiments, irradiated allogeneic MoDCs were cocultured with $\mathrm{CD}^{+} \mathrm{CD}_{45 \mathrm{RA}}{ }^{+} \mathrm{T}$ cells at a ratio of 1:10. After 5 days, stimulated T cells were expanded with 100 U/mL human recombinant IL-2 (Proleukin) (Roche, Basel, Switzerland) and analyzed for cytokine production between days 7 and 10 by intracellular staining and flow cytometry.

\section{Intracellular staining}

Expanded T cells were stimulated with $100 \mathrm{nM}$ phorbo myristate acetate and $1 \mu \mathrm{g} / \mathrm{mL}$ Ionomycin (both from Sigma) for 6 hours. During the last 4 hours of culture $10 \mu \mathrm{g} / \mathrm{mL}$ Brefeldin A (Sigma) was added. After fixation with $2 \%$ paraformaldehyde, $\mathrm{T}$ cells were permeabilized with phosphatebuffered saline containing $0.1 \%$ saponin, $2 \%$ fetal calf serum (FCS), and 5 mM EDTA; stained with fluorescein isothiocyanate (FITC)-labeled antiinterferon- $\gamma$ (anti-IFN- $\gamma$ ) and phycoerythrin (PE)-labeled anti-IL-4 (Becton Dickinson, Basel, Switzerland); and analyzed by flow cytometry.

\section{Fluorescence-activated cell sorter analysis of MoDCs}

MoDCs were analyzed on a FACScan flow cytometer (Becton Dickinson) after staining with the following monoclonal antibodies (mAbs): FITClabeled anti-CD83 (Immunotech, Berlin, Germany), FITC-labeled antiCD86, PE-labeled anti-CD80, anti-CCR5 (clone 2D7) (Pharmingen, Basel, Switzerland), anti-CCR1 (clone 53504.111), anti-CXCR4 (clone 12G5) (R\&D Systems, Wiesbaden-Nordenstadt, Germany), and anti-CCR7. ${ }^{13}$ As secondary reagents for unlabeled mAbs, FITC-conjugated sheep-antimouse (Silenus, Melbourne, Australia) and FITC-conjugated goat-antirat immunoglobulin-G (Jackson Immunoresearch, La Roche, Switzerland) were used.

\section{Cytokine assays}

Immature MoDCs were stimulated with a cocktail of proinflammatory cytokines (20 ng/mL TNF- $\alpha, 10 \mathrm{ng} / \mathrm{mL}$ IL-1 $\beta$, and $1000 \mathrm{U} / \mathrm{mL}$ IL-6), $500 \mathrm{ng}$ sCD40L (Immunex), or $20 \mu \mathrm{g} / \mathrm{mL}$ polyI:C (Sigma) in the presence or absence of $1 \mu \mathrm{g} / \mathrm{mL}$ PGE2. After 48 hours, culture supernatants were collected and analyzed by enzyme-linked immunosorbent assay (ELISA) by means of commercially available kits to detect IL-12p70 (Endogen, Woburn, MA), TNF- $\alpha$, and IL-10 (Pharmingen) according to the manufacturer's protocols.

\section{RNA isolation and complementary DNA synthesis}

Total RNA was isolated from immature and mature MoDCs by means of TRIZOL reagent (Invitrogen) according to the protocol provided by the manufacturer. We used $2 \mu \mathrm{g}$ total RNA for synthesis of first-strand complementary DNA (cDNA) by means of Moloney murine reverse transcriptase (Promega, Wallisellen, Switzerland) following the manufacturer's recommendations. The resulting cDNA was used for real-time reversetranscription polymerase chain reaction (RT-PCR).

\section{Real-time RT-PCR}

Real-time RT-PCR was performed by means of the LightCycler (Roche) and the LightCycler-DNA Master SYBR Green I kit (Roche) according to the protocol provided by the manufacturer. After initial denaturation for 30 seconds at $95^{\circ} \mathrm{C}$, thermal cycling was performed for 40 cycles with steps of $95^{\circ} \mathrm{C}$ for 5 seconds, $60^{\circ} \mathrm{C}$ for 10 seconds, and $72^{\circ} \mathrm{C}$ for 20 seconds, with the fluorescence being read at the end of each cycle. The following oligonucleotides were used as primers for the PCR: for CCR7, 5' -CCTGGGGAAACCAATGAAAAGC-3' and 5'-GAGCATGCCCACTGAAGAAGC-3'; for EP2, 5' -GCAATGCCTCCAATGACTCC-3' and 5'-GCGCCAGTGCCACCAGGG-3'; and for EP4, 5'-CACTCCCGGGGTCAATTCG-3' and 5'GGCATGGTTGATGGCCAGG-3'. Analysis was performed with Light 
Cycler Software 3. The obtained values were within the linear range of a standard curve and were normalized to yield the same amount of glyceraldehyde phosphate dehydrogenase (GAPDH) messenger RNA (mRNA). All PCR products were analyzed by determination of melting profiles as well as by agarose gel electrophoresis.

\section{Chemotaxis assay}

Chemotaxis of MoDCs was measured by migration through a polycarbonate filter with $5-\mu \mathrm{m}$ pore size in 24-well transwell chambers (Corning Costar, Cambridge, MA) with the use of AIM V as assay medium. Added to the lower chamber was $600 \mu \mathrm{L}$ assay medium containing $300 \mathrm{ng} / \mathrm{mL}$ CCL19 (R\&D Systems) or $250 \mathrm{ng} / \mathrm{mL}$ CCL21 (R\&D Systems), or assay medium alone as a control for spontaneous migration. We added $1 \times 10^{5}$ MoDCs unstimulated or stimulated for 48 hours with the indicated reagents to the upper chamber in a total volume of $100 \mu \mathrm{L}$ and incubated this for 3 hours at $37^{\circ} \mathrm{C}$. A $500-\mu \mathrm{L}$ aliquot of the cells that migrated to the bottom chamber was counted by flow cytometry acquiring events for a fixed time period of 60 seconds with the use of CellQuest software (Becton Dickinson). Each experiment was performed in duplicate. The mean number of spontaneously migrated cells was subtracted from the total number of migrated cells. Values are given as the mean number of migrated cells \pm SEM

\section{Results}

\section{Influence of PGE2 on phenotypical maturation and function} of MoDCs

In the first series of experiments, we investigated the effect of PGE2 on the phenotypic maturation and T-cell stimulatory functions of human MoDCs that were generated under serum-free conditions and stimulated with either sCD40L, polyI:C, or a cocktail of proinflammatory cytokines (TNF- $\alpha$, IL-1 $\beta$, and IL-6). ${ }^{24}$ Immature MoDCs were treated with each of the stimuli in the presence or absence of PGE2 for 48 hours, and the expression of maturation-related surface markers was analyzed by flow cytometry. Consistent with previous reports, ${ }^{24,26,27}$ PGE2 induced or up-regulated surface expression of CD83 and the costimulatory molecule CD80 on immature MoDCs and on MoDCs stimulated with the proinflammatory cytokines, but it had no effect on the phenotypic maturation of MoDCs treated with SCD40L or polyI:C (Figure 1). In contrast to MoDCs differentiated in the presence of FCS, our MoDCs, which were generated under serum-free conditions, displayed relatively high CD86 expression in the immature stage, and this was barely enhanced upon exposure to maturation stimuli (Figure 1, and data not shown).

The capability of immature MoDCs to stimulate allogeneic naive $\mathrm{CD}^{+}{ }^{+} \mathrm{T}$ cells was very low and increased when MoDCs displayed a mature phenotype (Figure 2). It is known that PGE2 together with TNF- $\alpha$, IL-6, and IL- $1 \beta$ increases the capacity of MoDCs to stimulate naive T cells, ${ }^{36}$ which we could confirm in our serum-free system. In addition, treatment of MoDCs with PGE2 alone improved their stimulatory capacity significantly, and to our surprise, PGE2 augmented the stimulatory functions of MoDCs matured in the presence of sCD40L and polyI:C as well (Figure 2B,D), although PGE2 had no effect on the phenotypic maturation of these MoDCs (Figure 1). These data suggest that PGE2, in addition to inducing the phenotypical maturation of DCs, also affects the function of these cells by promoting their T-cell stimulatory potential.

\section{PGE2 inhibits cytokine production by activated MoDCs}

As a consequence of maturation, MoDCs usually secrete inflammatory cytokines. ${ }^{37}$ PGE2 has previously been shown to inhibit the

\section{CD83 $\quad$ CD86 $\quad$ CD80}

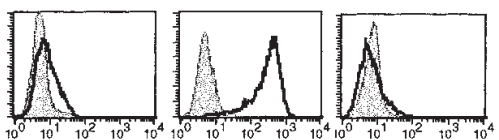

immature

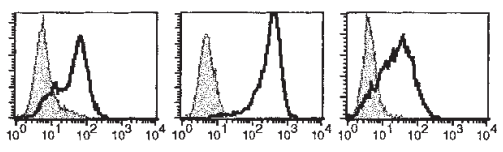

PGE2

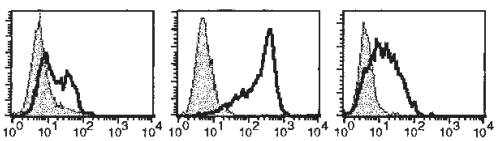

TNF- $\alpha$ IL-1B

IL-6

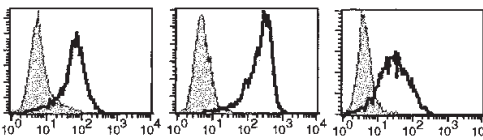

TNF- $\alpha$ IL- $1 \beta$

IL-6 PGE2
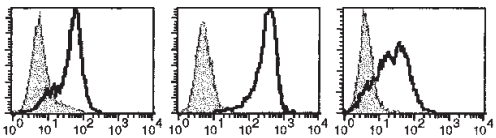

sCD40L

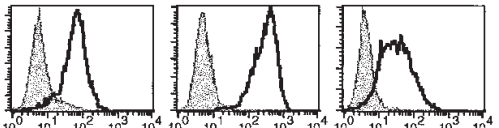

CD40L

PGE2

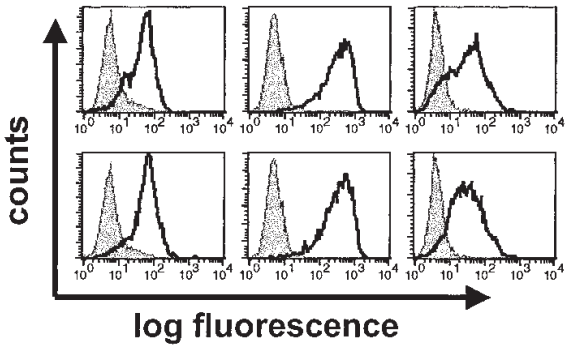

polyl:C

polyl:C

PGE2

Figure 1. Influence of PGE2 on the expression of CD83, CD86, and CD80 on immature and mature MoDCs. MoDCs were matured by treatment with a cocktail of proinflammatory cytokines (TNF- $\alpha$, IL-1 $\beta$, and IL-6), SCD40L, or polyl:C in the presence or absence of PGE2. After 48 hours, MoDCs were analyzed by flow cytometry for the expression of CD83, CD86, and CD80 (black lines). Filled gray histograms represent staining with isotype-matched irrelevant mAbs. The data shown are from 1 out of 8 experiments that all gave similar results.

production of bioactive IL-12p70 and TNF- $\alpha$, while enhancing the production of the immunosuppressive cytokine IL-10. ${ }^{28,38,39}$ To test these parameters in our experimental setup, we analyzed, by ELISA, the amount of IL-12p70, TNF- $\alpha$, and IL-10 present in the cell culture supernatant of MoDCs after 48 hours of stimulation with the respective stimuli (Figure 3). Considerable amounts of IL-12p70 could be detected in the supernatant of polyI:Cstimulated MoDCs, but interestingly, PGE2 down-regulated its expression by more than $50 \%$ (Figure 3A). In contrast, treating cells with $500 \mathrm{ng} / \mathrm{mL}$ sCD40L or the cocktail of proinflammatory cytokines did not alter IL-12p70 secretion. However, when we raised the concentration of sCD40L to 2 to $3 \mu \mathrm{g} / \mathrm{mL}$ for stimulation, MoDCs produced levels of IL-12p70 similar to what we observed with polyI:C (data not shown). The addition of PGE2 decreased the secretion of TNF- $\alpha$ by MoDCs that were stimulated with polyI:C or sCD40L, while immature MoDCs did not produce detectable amounts of $\mathrm{TNF}-\alpha$ at any time point (Figure 3B). Because TNF- $\alpha$ was present in the cytokine cocktail used for MoDC maturation, the quantities of secreted TNF- $\alpha$ could not be determined in this case. In agreement with previous reports, ${ }^{39}$ IL-10 production was significantly increased in immature MoDCs 
A

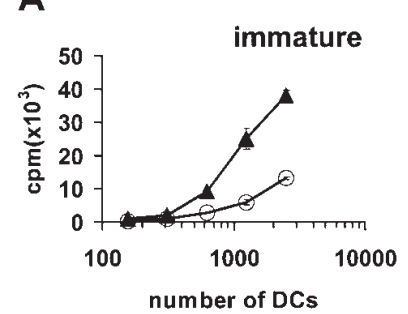

C TNF- $\alpha$ IL-1 $\beta$ IL-6

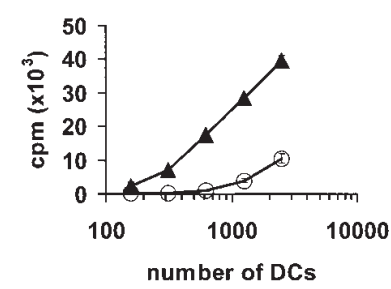

Figure 2. Enhancement of T-cell stimulatory capacity by PGE2. Naive $\mathrm{CD} 4{ }^{+} \mathrm{CD} 45 \mathrm{RA}{ }^{+} \mathrm{T}$ cells were incubated with graded numbers of allogeneic MoDCs that had been either left untreated $(A)$ or stimulated with polyl:C (B), a cocktail of proinflammatory cytokines (TNF- $\alpha, \mathrm{IL}-1 \beta$, and IL-6) (C), or SCD40L (D) in the presence (triangles) or absence (open circles) of PGE2. After 4 days of coculture, the proliferative response was measured by ${ }^{3} \mathrm{H}$-thymidine incorporation. The data are shown as the mean of duplicate cultures \pm SEM and represent 1 experiment out of 4 yielding similar results.

treated with PGE2 alone. On the contrary, PGE2 did not affect IL-10 production in MoDCs in combination with sCD40L or proinflammatory cytokines, while it decreased IL-10 secretion by about 3-fold in MoDCs stimulated with polyI:C (Figure 3C). Taken together, these data suggest that PGE2 generally suppresses the production of the examined cytokines in mature MoDCs, but stimulates immature MoDCs to produce IL-10.

\section{T-cell priming and polarization are not affected by PGE2}

PolyI:C-matured MoDCs have been shown to be strong inducers of $\mathrm{T}_{\mathrm{H}} 1$ differentiation, ${ }^{3}$ which is, at least in part, due to the secretion of bioactive IL-12p70. Since PGE2 suppressed IL-12p70 production in polyI:C-treated MoDCs (Figure 3A), we next asked whether adding PGE2 would affect $\mathrm{T}_{\mathrm{H}} 1$ polarization. Naive CD4 ${ }^{+}$ $\mathrm{T}$ cells were primed with allogeneic MoDCs that had been stimulated with polyI:C in the presence or absence of PGE2. After 7 to 10 days, the pattern of IFN- $\gamma$ and IL-4 production by these T cells was analyzed by flow cytometry after intracellular cytokine staining. Our data suggest that PGE2 had no effect on the $\mathrm{T}_{\mathrm{H}} 1$-type polarizing capacities of MoDCs irrespective of whether they were matured with polyI:C (Figure 4) or any other stimulus tested that led comparable numbers of $\mathrm{CD}^{+}{ }^{+} \mathrm{T}$ cells to express IL-4 (2\% to $4 \%$ ) or IFN- $\gamma$ (17\% to $25 \%$ ) (data not shown).

\section{PGE2 modulates chemokine receptor expression on MoDCs}

Maturation of DCs is also accompanied by down-regulation of inflammatory chemokine receptors and induction of CCR7. ${ }^{10}$ This change is essential for migration of DCs to lymphoid organs and optimal initiation of an immune response. Hence, we analyzed the effect of PGE2 on surface expression of the chemokine receptors CCR1, CCR5, CXCR4, and CCR7 on MoDCs generated under serum-free conditions (Figure 5). Unexpectedly, CCR1 expression was present only on freshly isolated monocytes, and after 8 days of cultivation, it was detectable neither in medium containing GMCSF and IL-4 nor in the presence of any maturing agent tested (data
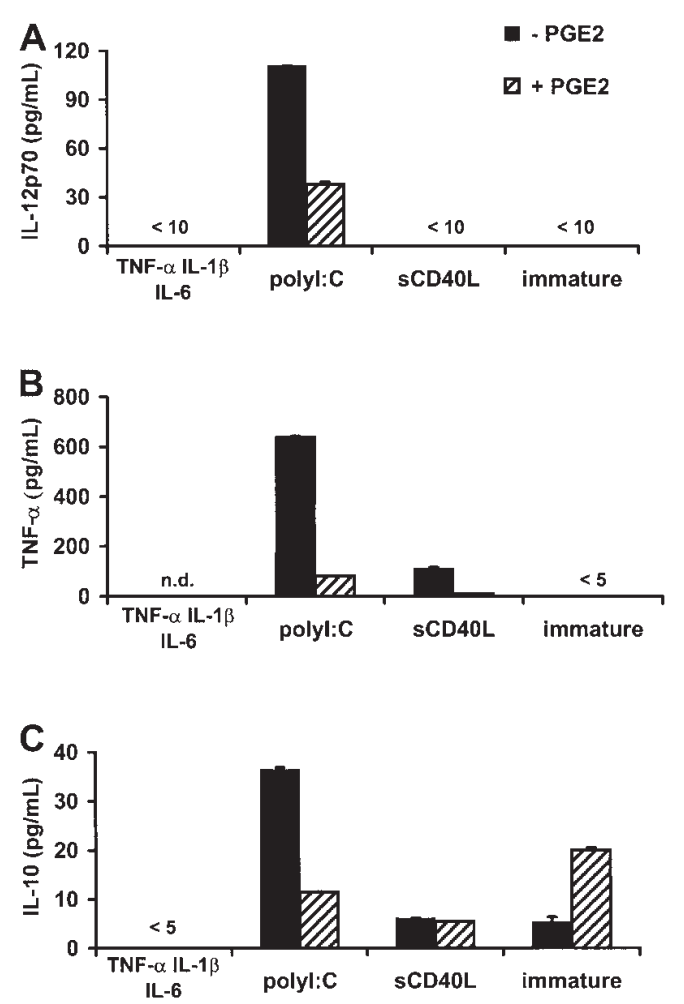

Figure 3. PGE2 inhibition of cytokine production by activated MoDCs. Immature MoDCs were stimulated with a cocktail of proinflammatory cytokines (TNF- $\alpha$, IL-1 $\beta$, and IL-6); SCD40L, or polyl:C in the presence (hatched bars) or absence of PGE2 (black bars), and ELISA assays were performed with culture supernatants collected after 48 hours. The detection limit was $10 \mathrm{pg} / \mathrm{mL}$ for IL-12p70 (A) and $5 \mathrm{pg} / \mathrm{mL}$ for TNF- $\alpha$ (B) and IL-10 (C). The amount of TNF- $\alpha$ was not determined in the culture supernatant of MoDCs matured with a cocktail of proinflammatory cytokines because TNF- $\alpha$ was included in the cocktail. The data are shown as the mean of duplicate measurements \pm SEM and are from 1 representative experiment out of 3 performed.

not shown). Immature MoDCs expressed relatively high levels of CCR5 but undetectable levels of CXCR4 and CCR7. The addition of sCD40L or polyI:C resulted in slight down-regulation of CCR5, whereas the proinflammatory cytokines did not have such an effect. In the presence of PGE2, however, CCR5 was further downregulated on both immature and mature MoDCs. An up-regulation of CXCR4 was barely visible on MoDCs matured with sCD40L and polyI:C, and CXCR4 expression was negligible on MoDCs stimulated with the proinflammatory cytokines. In the presence of PGE2, however, CXCR4 expression was increased on immature and mature MoDCs. Even more prominent was the effect of PGE2 on CCR7 expression, when PGE2 was added to untreated immature MoDCs or to MoDCs treated with the cocktail of proinflammatory cytokines (Figure 5). As expected, CCR7 was induced in MoDCs by sCD40L and polyI:C. While PGE2 did not significantly
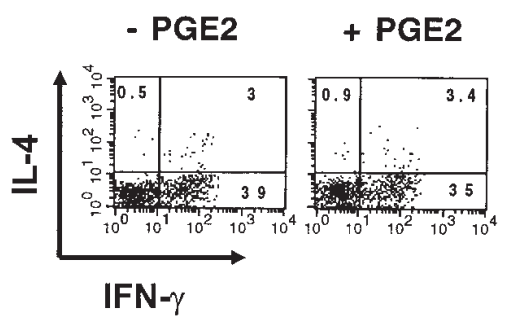

Figure 4. Lack of significant PGE2 effect on T-cell polarization. MoDCs matured by treatment with polyl:C, with or without PGE2, were used to stimulate allogeneic CD4 ${ }^{+}$CD 45RA + T cells. After 7 to 10 days, IFN- $\gamma$ and IL-4 production of the polyclonal $\mathrm{T}$-cell lines was analyzed by flow cytometry after intracellular staining. 
CCR5 CXCR4 CCR7

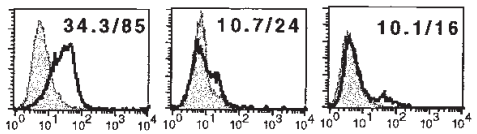

immature
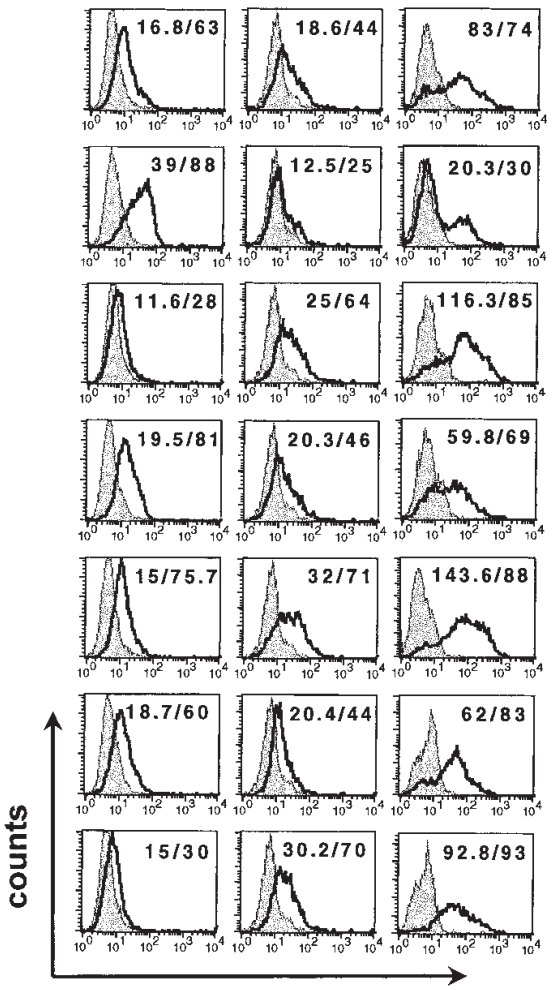

log fluorescence

Figure 5. PGE2 modulation of chemokine receptor expression on MoDCs. MoDCs were matured by treatment with a cocktail of proinflammatory cytokines (TNF- $\alpha$, IL-1 $\beta$, and IL-6), SCD40L, or polyl:C in the presence or absence of PGE2. After 48 hours, MoDCs were analyzed by flow cytometry for surface expression of the chemokine receptors CCR5, CXCR4, and CCR7 (black lines). Filled gray histograms represent staining with isotype-matched irrelevant mAbs. The inserted numbers resemble the mean fluorescence intensity by the percentage of positive cells. The data shown are from 1 out of 6 experiments that gave similar results.

up-regulate CCR7 expression on polyI:C-treated MoDCs, it was able to further enhance CCR7 expression in combination with sCD40L.

Surface expression of CCR7 correlated well with CCR7 mRNA levels in these cells as determined by real-time RT-PCR (Figure 6). Marginal expression of CCR7 mRNA was detected in immature and cytokine-stimulated MoDCs. In MoDCs stimulated with sCD40L and polyI:C, the CCR7 mRNA expression was upregulated 20- and 45-fold, respectively, as compared with unstimulated cells. With additional stimulation by PGE2, the expression of CCR7 mRNA increased 38-fold in cytokine-stimulated, 49-fold in sCD40L-activated, and 52-fold in polyI:C-treated MoDCs when compared with immature MoDCs.

\section{PGE2 is required for efficient migration of MoDCs toward CCL19 and CCL21}

As we could detect up-regulation of CCR7 surface expression on MoDCs by the action of PGE2 during maturation, we next analyzed whether this functionally results in a better migration of MoDCs toward the 2 known ligands for CCR7: CCL19 and CCL21. 40,41 Therefore, immature MoDCs and MoDCs stimulated

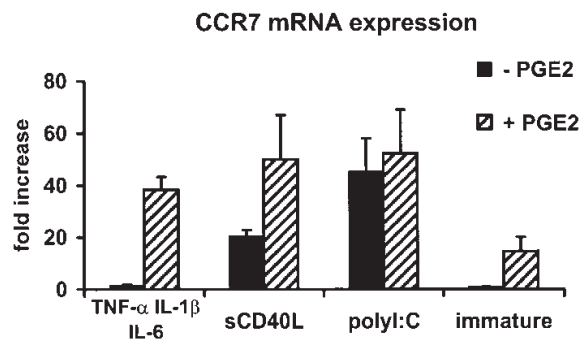

Figure 6. PGE2 enhancement of CCR7 mRNA expression in MoDCs. Total RNA was isolated from immature and mature MoDCs that had been stimulated with a cocktail of proinflammatory cytokines (TNF- $\alpha$, IL-1 $\beta$, and IL-6), SCD40L, or polyl:C in the presence or absence of PGE2 for 48 hours. The expression of CCR7 mRNA was analyzed by real time RT-PCR. After standardization to yield the same amount of GAPDH mRNA, all samples were compared with immature MoDCs (value $=1$ ). The data shown represent the mean CCR7 mRNA level \pm SEM obtained from 3 independent experiments with MoDC preparations from different donors.

with sCD40L, polyI:C, or the cytokine cocktail, in the absence or presence of PGE2, were examined in a transwell migration assay (Figure 7). In accordance with undetectable CCR7 surface expression, immature MoDCs and MoDCs that were matured in the presence of proinflammatory cytokines did not migrate at all or barely migrated in response to CCL19 and CCL21, respectively. Interestingly, when CCR7 surface expression was up-regulated by PGE2, immature MoDCs also gained a moderate capability to migrate toward CCL19 and CCL21, while the migratory response of MoDCs matured with proinflammatory cytokines was induced about 80-fold for CCL19 and at least 6-fold for CCL21. To our surprise, MoDCs matured by sCD40L and polyI:C exhibited only weak migratory activity in response to both CCR7 ligands, although they expressed comparable levels of CCR7 on their surface. Costimulation with PGE2, however, also substantially amplified the capability of these cells to migrate toward CCL19 as
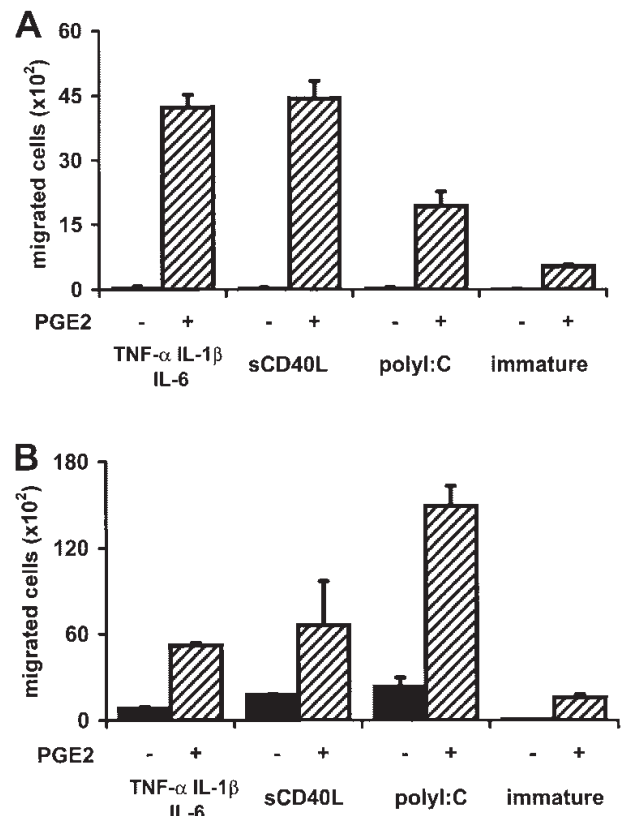

Figure 7. Need for PGE2 for efficient migration of MoDCs toward CCL19 and CCL21. MoDCs were matured by treatment with a cocktail of proinflammatory cytokines (TNF- $\alpha$, IL-1 $\beta$, and IL-6), SCD40L, or polyl:C in the presence or absence of PGE2 for 48 hours. Unstimulated and stimulated MoDCs were analyzed for their chemotactic response to CCL19 (panel A) or CCL21 (panel B). The data are shown as the mean of duplicate cultures \pm SEM and are from 1 representative experiment out of 3 performed. The mean number of spontaneously migrated cells were subtracted from the number of cells that migrated in response to chemokines. 
well as CCL21. Taken together, these experiments show that MoDCs require a maturation stimulus and PGE2-provided signals to acquire the potential to migrate effectively in response to the lymph node-derived chemokines CCL19 and CCL21.

\section{PGE2 receptors EP2 and EP4 are expressed in MoDCs and mediate the increased migratory potential of MoDCs}

Binding of PGE2 to its receptors can trigger quite distinct signals in cells because, while the stimulation of the prostaglandin receptors EP1 and EP3 leads to intracellular mobilization of $\mathrm{Ca}^{++}$and decreased cAMP levels, the $2 \mathrm{G}_{\mathrm{s}}$-coupled receptors EP2 and EP4 signal through increased cAMP by stimulation of the adenylate cyclase. ${ }^{33}$ First, we quantitatively investigated mRNA expression of the 2 prostaglandin receptors reported to be expressed in monocytes and peritoneal macrophages, EP2 and EP4, ${ }^{34,35}$ in immature or mature MoDCs by real time RT-PCR. As shown in Figure 8A, the expression of EP2 mRNA was not significantly altered by the different maturation stimuli. However, in the presence of PGE2, EP2 mRNA expression was down-regulated in all cases, most prominently in immature and sCD40L-stimulated MoDCs. In contrast, EP4 mRNA was up-regulated by the different maturation stimuli; the up-regulation was most evident for sCD40L, followed by polyI:C, and was least prominent for proinflammatory cytokines (Figure 8B). Treatment with PGE2 likewise resulted in a markedly reduced expression of EP4 mRNA in all samples. To test the expression of EP1 and EP3 mRNA, we performed RT-PCR on the same cells, but no EP1 and EP3 mRNAs were detected, indicating that these 2 types of prostaglandin receptors are not expressed in MoDCs (data not shown).

These data suggest that the effects of PGE2 on MoDCs are probably mediated by elevating the intracellular second messenger, cAMP, via signaling through EP2 and EP4. To confirm this notion, we investigated whether forskolin, a pharmacological activator of adenylate cyclase; cholera toxin, which activates the $\mathrm{G} \alpha_{\mathrm{s}}$ subunit of $G$ proteins and therefore mimics signaling of $\mathrm{G}_{\mathrm{s}}$-coupled receptors like EP2 and EP4; or 11-deoxy-PGE1, a synthetic analog of PGE1 and specific agonist for EP2 and EP4, exerts the same effect as PGE2 on the migratory behavior of MoDCs stimulated with polyI:C. As expected, any of these agents was able to significantly enhance the migratory capacity of matured MoDCs in response to CCL21 and CCL19 (Figure 8C, and data not shown), thus replacing the requirement for PGE2. Additionally, putative signaling via EP1 and EP3 was blocked by adding $50 \mathrm{ng} / \mathrm{mL}$ pertussis toxin, alone or in combination with PGE2, since, unlike EP2 and EP4, most of the intracellular effects by these receptors are coupled to pertussis toxin-sensitive $\mathrm{G}_{\mathrm{i}}$-proteins. As shown in Figure 8D, the addition of pertussis toxin did not inhibit the PGE2-induced upregulation of CCR7 surface expression in MoDCs matured in the presence of proinflammatory cytokines, indicating that signaling through $\mathrm{G}_{\mathrm{i}}{ }^{-}$ proteins is not required for the effects mediated by PGE2. Due to coupling of CCR7 to $\mathrm{G}_{\mathrm{i}}$-proteins, however, the migratory response of pertussis toxin-treated mature MoDCs was completely inhibited (Figure 8C).

\section{Discussion}

CCR7 expression on mature DCs is essential for their homing to secondary lymphoid organs where the CCR7 ligands CCL19 and CCL21 are produced. ${ }^{13}$ Our results show that PGE2 induces CCR7 expression in MoDCs matured with proinflammatory cytokines and enhances CCR7 expression on MoDCs stimulated with sCD40L,
A

EP2 mRNA expression
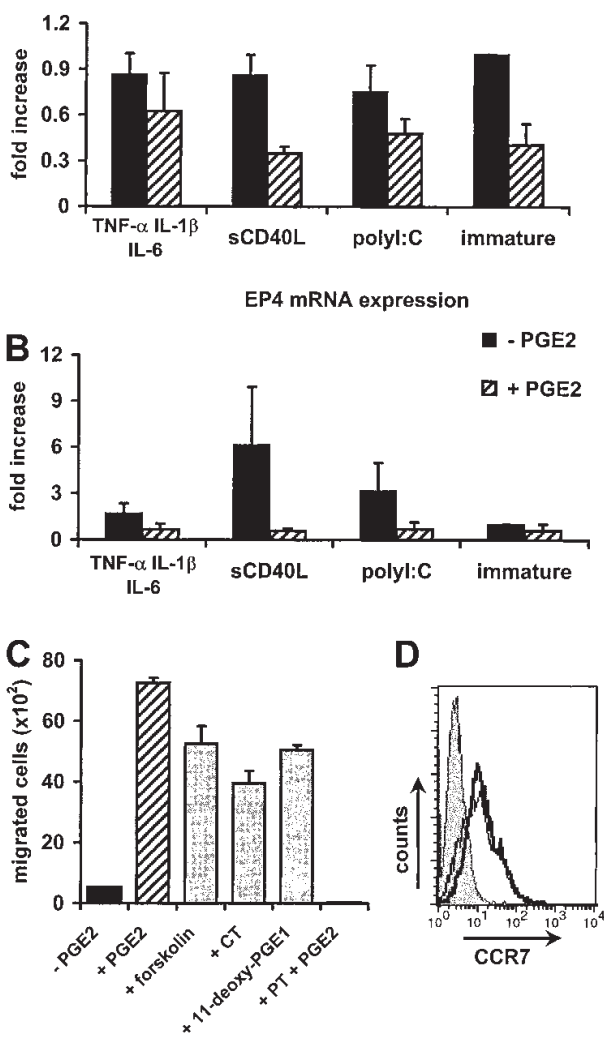

D

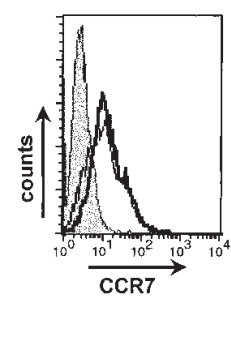

Figure 8. EP2 and EP4 expression of prostaglandin receptors in MoDCs. (A-B) Total RNA was isolated from immature and mature MoDCs that had been stimulated with a cocktail of proinflammatory cytokines (TNF- $\alpha$, IL-1 $\beta$, and IL-6), SCD40L, or polyl: $C$ in the presence or absence of PGE2 for 48 hours. The expression of EP2 (A) and EP4 (B) mRNA was analyzed by real time RT-PCR. After standardization to yield the same amount of GAPDH mRNA, all samples were compared with immature MoDCs (value $=1$ ). The data shown represent the mean EP2 or EP4 mRNA level \pm SEM obtained from 3 independent experiments with MoDC preparations from different donors. (C) MoDCs were stimulated for 48 hours with polyl:C alone (-PGE2), with polyl:C in combination with PGE2; or with polyl:C along with forskolin, cholera toxin, 11-deoxy-PGE1, or pertussis toxin in combination with PGE2; the MoDCs were subsequently analyzed in a transwell migration assay for their chemotactic response to CCL21. (D) MoDCs stimulated for 48 hours with TNF- $\alpha$, IL-1 $\beta$, IL-6, and PGE2 alone (thin line), or along with pertussis toxin (bold line), were analyzed for CCR7 surface expression by flow cytometry. The filled gray histogram represents staining with an isotype-matched irrelevant mAb. The data shown in panels $C$ and $D$ are from 1 out of 3 experiments that gave similar results.

but has no additional effect on CCR7 expression of MoDCs matured with polyI:C. However, the migratory capability of MoDCs stimulated with sCD40L and poyI:C in the absence of PGE2 seems to be impaired, since these cells did not effectively migrate in response to the 2 CCR7 ligands CCL19 and CCL21. In our serum-free system, signals provided by PGE2 were absolutely required for an efficient migration of mature MoDCs in response to CCL19 and CCL21. These data, as well as observations made by Luft et al (Eugene Maraskovsky, written communication, September 2001) that PGE2 also positively affects migration of MoDCs toward the CXCR4 ligand CXCL12 (stromal cell derived factor-1), imply that the migratory capacity of MoDCs toward constitutively expressed chemokines in general is selectively promoted by the action of PGE2.

D'Amico et $\mathrm{al}^{42}$ have recently shown that IL-10-treated MoDCs and monocytes did not migrate in response to CCL3, CCL4, or CCL5 despite CCR1 and CCR5 surface expression. Moreover, it was demonstrated that in the presence of IL-10, MoDCs had a defective capacity for signaling through chemokine receptors, thus 
indicating that they were uncoupled through IL-10. Similarly, activation of CCR7 receptors expressed on mature MoDCs might be inhibited by an as-yet-unidentified mechanism in the absence of signals provided by PGE2 during maturation. CCR7 receptor uncoupling was also reported for plasmacytoid DCs isolated from peripheral blood, which express quite high levels of CCR7 on their surface but fail to migrate in response to CCL19 unless they received a maturation stimulus that restored their migratory capacity. ${ }^{43}$ Although it seems very unlikely, we should point out that as a hypothetical alternative to the coupling of CCR7 to signaling, the effect of PGE2 on MoDC migration toward CCR7 ligands might be due to the induction or activation of an as-yetunknown chemokine receptor for CCL19 and CCL21.

The effect of PGE2 was mediated mainly by the intracellular second messenger, cAMP, because only prostaglandin receptors EP2 and EP4, which signal through increased cAMP, were expressed in MoDCs and were down-regulated after PGE2 treatment. Moreover, the EP2/EP4 agonist 11-deoxy-PGE1, as well as cAMP-elevating agents like forskolin or cholera toxin, could substitute for PGE2 in the activation of MoDC migration toward lymph node-derived chemokines. Signals mediated by intracellular cAMP were shown to be involved in cell polarization and uropod formation induced by chemokines. ${ }^{44}$ In particular, activation of the cAMP-dependent protein kinase (PKA) seems to be important, as the inhibition of PKA led to an abrogation of the chemokinemediated uropod formation in T cells. ${ }^{45}$ Furthermore, upregulation of metalloproteinases, which are involved in migration processes, is mediated by elevated intracellular cAMP. ${ }^{46}$

One reason why exogenous PGE2 is required to fully restore the migratory capacity of MoDCs in response to CCL19 and CCL21 could be an impaired production of PGE2 by MoDCs generated under serum-free conditions in the presence of GM-CSF and IL-4. At sites of inflammation, the formation of prostaglandins from its precursor, arachidonic acid, is catalyzed by the prostaglandin synthetase cyclo-oxigenase 2 (COX-2) that is induced in monocytes by inflammatory stimuli, such as bacterial lipopolysaccharide (LPS) or proinflammatory cytokines (TNF- $\alpha$, IL-1 $\beta$, and IL-6), ${ }^{47}$ but is suppressed in the presence of IL-4. ${ }^{48}$ Moreover, it has been shown that IL-4 also inhibits cytosolic phospholipase A2, which is required for the liberation of the prostaglandin precursor arachidonic acid from membrane phospholipids. ${ }^{48,49}$ Although MoDCs are reportedly resistant to IL-4-mediated down-regulation of COX-2,50 a limitation in the substrate for inducible COX-2 may nonetheless lead to decreased production of PGE2 in MoDCs generated in the presence of IL-4. Inhibition of cytosolic phospholipase A2 by IL-4 also affects the formation of other arachidonic acid metabolites, such as leukotrienes, that have been shown to play an important role in migration of DCs toward CCL19 as well. ${ }^{14}$

This could also explain why the defective migratory capacity of MoDCs toward lymph node-derived chemokines remained unnoticed in previous studies in which MoDCs were generated in the presence of GM-CSF and IL-13 instead of IL-4 or in cell culture medium supplemented with FCS.8,51 However, Luft et al (Eugene Maraskovsky, written communication, September 2001) showed that MoDCs generated in the presence of FCS display only moderate migratory responses toward CCL19 and CCL21, but that this was significantly enhanced in the presence of PGE2.

It has been shown that the chemokine receptors CCR1 and CCR5, which respond to the inflammatory chemokines CCL3, CCL4, and CCL5, are down-regulated on the surface of MoDCs owing to an autocrine mechanism. ${ }^{10}$ Probably because of different cultivation conditions, we were unable to detect CCR1 surface expression on immature MoDCs generated in a serum-free environment. PGE2 has been previously shown to down-regulate CCR5 on human monocytes, thus inducing resistance to human immunodeficiency virus- 1 infection. ${ }^{52,53}$ Analogously to monocytes, an almost complete down-regulation of CCR5 surface expression on MoDCs could be observed only in the presence of PGE2 (Figure 5).

In agreement with previous reports, ${ }^{24,26,27}$ we demonstrated that PGE2 elevated maturation-related surface markers and enhanced the T-cell stimulatory capacity of MoDCs matured with proinflammatory cytokines. The latter positive effect of PGE2 on MoDCs was not restricted to cytokine-stimulated MoDCs but was also valid for MoDCs activated with sCD40L or polyI:C, thus suggesting that PGE2 is a key promoter of MoDC function.

The presence of PGE2 during the maturation of MoDCs decreased the secretion of bioactive IL-12p70 by MoDCs stimulated with polyI:C, in accordance with previous reports. ${ }^{26,54}$ Interestingly, in LPS-treated macrophages, the EP4 receptor is apparently involved in the down-regulation of TNF- $\alpha$ and IL-12 secretion, ${ }^{55}$ suggesting that the down-regulation of TNF- $\alpha$ and IL-12p70 production that we found in human MoDCs could be based on the activation of EP4.

Kalinski et $\mathrm{al}^{56}$ found that the presence of PGE2 during maturation yielded MoDCs, which promoted $\mathrm{T}_{\mathrm{H}} 2$ differentiation. Our results are contradictory to this finding, because we could not see an effect of PGE2 on $\mathrm{T}_{\mathrm{H}}$-cell polarization by MoDCs in any of our experiments (Figure 4, and data not shown). Also, Steinbrink et $\mathrm{al}^{36}$ demonstrated that naive $\mathrm{T}_{\mathrm{H}}$ cells primed by MoDCs and matured in the presence of proinflammatory cytokines (TNF- $\alpha$, IL-1 $\beta$, and IL-6) and PGE2, displayed a $\mathrm{T}_{\mathrm{H}} 1$ rather than a $\mathrm{T}_{\mathrm{H}} 2$ cytokine profile in accordance with our data. As discussed previously, ${ }^{36}$ one reason for this discrepancy could be different culture conditions for the generation of MoDCs, resulting in distinct populations, as well as varying DC-to-T-cell ratios used for $\mathrm{T}_{\mathrm{H}}$-cell priming.

In conclusion, signals provided by the action of PGE2 selectively ameliorate functional maturation of MoDCs generated under serum-free conditions, allowing them to efficiently prime $\mathrm{T}_{\mathrm{H}}$ cells and migrate in response to CCL19 and CCL21. This is an important new parameter that needs to be considered for the in vitro differentiation of MoDCs as cellular vaccines in cancer immunotherapy.

\section{Acknowledgements}

We thank Immunex Corporation for providing sCD40L and Annalisa Macagno for critical reading of the manuscript and help with ELISA experiments. Wolfhart Seelentag as well as Hans Schiefer, and Markus Arn are acknowledged for the irradiation of cells and Dr Markus Fopp and the personnel of St Gallen blood bank for supplying blood products.

\section{References}

1. Banchereau J, Steinman RM. Dendritic cells and the control of immunity. Nature. 1998:392:245252

2. Langenkamp A, Messi M, Lanzavecchia A, Sallusto F. Kinetics of dendritic cell activation: impact on priming of $\mathrm{TH} 1, \mathrm{TH} 2$ and nonpolarized T cells. Nat Immunol. 2000;1:311-316.

3. Cella M, Salio M, Sakakibara $Y$, Langen $\mathrm{H}$, Julkunen I, Lanzavecchia A. Maturation, activation, and protection of dendritic cells induced by double-stranded RNA. J Exp Med. 1999;189:821829.

4. Gagliardi MC, Sallusto F, Marinaro M, Langenkamp A, Lanzavecchia A, De Magistris MT. Cholera toxin induces maturation of human dendritic 
cells and licences them for Th2 priming. Eur J Immunol. 2000;30:2394-2403.

5. Sallusto F, Cella M, Danieli C, Lanzavechia A Dendritic cells use macropinocytosis and the mannose receptor to concentrate macromolecules in the major hisotcompatibility complex class II compartment: downregulation by cytokines and bacterial products. J Exp Med. 1995; 182:389-400.

6. Cella M, Engering A, Pinet V, Pieters J, Lanzavecchia $A$. Inflamatory stimuli induce accumulation of MHC class II complexes on dendritic cells. Nature. 1997;388:782-787.

7. Rescigno M, Citterio S, Thery C, et al. Bacteriainduced neo-biosynthesis, stabilization, and surface expression of functional class I molecules in mouse dendritic cells. Proc Natl Acad Sci U S A. 1998;95:5229-5234.

8. Sallusto F, Palermo B, Lenig D, et al. Distinct patterns and kinetics of chemokine production regulate dendritic cell function. Eur J Immunol. 1999; 29:1617-1625

9. Springer TA. Traffic signals for lymphocyte recirculation and leukocyte emigration: the multistep paradigm. Cell. 1994;76:301-314

10. Sallusto F, Schaerli $P$, Loetscher $P$, et al. Rapid and coordinated switch in chemokine receptor expression during dendritic cell maturation. Eur J Immunol. 1998;28:2760-2769.

11. Cyster JG. Chemokines and cell migration in secondary lymphoid organs. Science. 1999;286: 2098-2102.

12. Charbonnier AS, Kohrgruber N, Kriehuber E, Stingl G, Rot A, Maurer D. Macrophage inflammatory protein 3alpha is involved in the constitutive trafficking of epidermal langerhans cells. J Exp Med. 1999;190:1755-1768.

13. Forster $R$, Schubel A, Breitfeld D, et al. CCR7 coordinates the primary immune response by establishing functional microenvironments in secondary lymphoid organs. Cell. 1999;99:23-33.

14. Robbiani DF, Finch RA, Jager D, Muller WA, Sartorelli AC, Randolph GJ. The leukotriene C(4) transporter MRP1 regulates CCL19 (MIP-3beta, ELC)-dependent mobilization of dendritic cells to lymph nodes. Cell. 2000;103:757-768.

15. Randolph GJ. Dendritic cell migration to lymph nodes: cytokines, chemokines, and lipid mediators. Semin Immunol. 2001;13:267-274.

16. Moodycliffe AM, Shreedhar V, Ullrich SE, et al. CD40-CD40 ligand interactions in vivo regulate migration of antigen-bearing dendritic cells from the skin to draining lymph nodes. J Exp Med. 2000;191:2011-2020.

17. Sallusto F, Lanzavecchia A. Efficient presentation of soluble antigen by cultured human dedritic cells is maintained by granulocyte/macrophage colony-stimulating factor plus interleukin 4 and downregulated by tumor necrosis factor $\alpha$. J Exp Med. 1994;179:1109-1118.

18. Peters $\mathrm{JH}, \mathrm{Xu} \mathrm{H}$, Ruppert J, Ostermeier D, Friedrichs D, Gieseler RK. Signals required for differentiating dendritic cells from human monocytes in vitro. Adv Exp Med Biol. 1993;329:275-280.

19. Morse MA, Coleman RE, Akabani G, Niehaus N, Coleman D, Lyerly HK. Migration of human dendritic cells after injection in patients with metastatic malignancies. Cancer Res. 1999;59:56-58.

20. Jonuleit H, Schmitt E, Schuler G, Knop J, Enk $\mathrm{AH}$. Induction of interleukin 10-producing, nonproliferating $\mathrm{CD} 4(+) \mathrm{T}$ cells with regulatory properties by repetitive stimulation with allogeneic immature human dendritic cells. J Exp Med. 2000; 192:1213-1222.

21. Dhodapkar MV, Steinman RM, Krasovsky J, Munz C, Bhardwaj N. Antigen-specific inhibition of effector T cell function in humans after injection of immature dendritic cells. J Exp Med. 2001;193:233-238.

22. Thurner B, Haendle I, Roder C, et al. Vaccination with mage-3A1 peptide-pulsed mature, monocyte-derived dendritic cells expands specific cytotoxic $T$ cells and induces regression of some me- tastases in advanced stage IV melanoma. J Exp Med. 1999;190:1669-1678.

23. Rieser C, Ramoner R, Holtl L, et al. Mature dendritic cells induce T-helper type-1-dominant immune responses in patients with metastatic renal cell carcinoma. Urol Int. 1999;63:151-159.

24. Jonuleit $\mathrm{H}$, Kuhn $\mathrm{U}$, Muller $\mathrm{G}$, et al. Pro-inflammatory cytokines and prostaglandins induce maturation of potent immunostimulatory dendritic cells under fetal calf serum-free conditions. Eur J Immunol. 1997;27:3135-3142.

25. Tilley SL, Coffman TM, Koller BH. Mixed messages: modulation of inflammation and immune responses by prostaglandins and thromboxanes. J Clin Invest. 2001:108:15-23.

26. Kalinski P, Schuitemaker JH, Hilkens CM Kapsenberg ML. Prostaglandin E2 induces the final maturation of IL-12-deficient CD1a+CD83+ dendritic cells: the levels of IL-12 are determined during the final dendritic cell maturation and are resistant to further modulation. J Immunol. 1998 161:2804-2809.

27. Rieser C, Bock G, Klocker H, Bartsch G, Thurnher M. Prostaglandin E2 and tumor necrosis factor alpha cooperate to activate human dendritic cells: synergistic activation of interleukin 12 production. J Exp Med. 1997;186:1603-1608.

28. Kalinski P, Vieira PL, Schuitemaker JH, de Jong EC, Kapsenberg ML. Prostaglandin E(2) is a selective inducer of interleukin-12 p40 (IL-12p40) production and an inhibitor of bioactive IL-12p70 heterodimer. Blood. 2001;97:3466-3469.

29. Grufman P, Karre K. Innate and adaptive immunity to tumors: IL-12 is required for optimal responses. Eur J Immunol. 2000;30:1088-1093.

30. Nishimura T, Nakui M, Sato M, et al. The critical role of Th1-dominant immunity in tumor immunology. Cancer Chemother Pharmacol. 2000;46 (suppl):S52-S61.

31. Schuler T, Qin Z, Ibe S, Noben-Trauth N, Blankenstein $\mathrm{T}$. T helper cell type 1-associated and cytotoxic $\mathrm{T}$ lymphocyte-mediated tumor immunity is impaired in interleukin 4-deficient mice. J Exp Med. 1999;189:803-810.

32. Coleman RA, Smith WL, Narumiya S. International Union of Pharmacology classification of prostanoid receptors: properties, distribution, and structure of the receptors and their subtypes. Pharmacol Rev. 1994;46:205-229.

33. Breyer RM, Bagdassarian CK, Myers SA, Breyer $M D$. Prostanoid receptors: subtypes and signaling. Annu Rev Pharmacol Toxicol. 2001;41:661-690.

34. Meja KK, Barnes PJ, Giembycz MA. Characterization of the prostanoid receptor(s) on human blood monocytes at which prostaglandin E2 inhibits lipopolysaccharide-induced tumour necrosis factor-alpha generation. Br J Pharmacol. 1997; 122:149-157.

35. Ikegami R, Sugimoto $Y$, Segi E, et al. The expression of prostaglandin E receptors EP2 and EP4 and their different regulation by lipopolysaccharide in $\mathrm{C} 3 \mathrm{H} / \mathrm{HeN}$ peritoneal macrophages. $\mathrm{J} \mathrm{Im}$ munol. 2001;166:4689-4696.

36. Steinbrink K, Paragnik L, Jonuleit $H$, Tuting $T$, Knop J, Enk AH. Induction of dendritic cell maturation and modulation of dendritic cell-induced immune responses by prostaglandins. Arch Dermatol Res. 2000;292:437-445.

37. Sallusto F, Lanzavecchia A. Mobilizing dendritic cells for tolerance, priming, and chronic inflammation. J Exp Med. 1999;189:611-614.

38. Strassmann G, Patil-Koota V, Finkelman F, Fong $\mathrm{M}$, Kambayashi T. Evidence for the involvement of interleukin 10 in the differential deactivation of murine peritoneal macrophages by prostaglandin E2. J Exp Med. 1994;180:2365-2370.

39. Kambayashi T, Alexander HR, Fong M, Strassmann G. Potential involvement of IL-10 in suppressing tumor-associated macrophages: colon26-derived prostaglandin E2 inhibits TNF-alpha release via a mechanism involving IL-10. J Immunol. 1995;154:3383-3390.
40. Yoshida R, Imai T, Hieshima K, et al. Molecular cloning of a novel human CC chemokine EBI1ligand chemokine that is a specific functional ligand for EBI1, CCR7. J Biol Chem. 1997;272: 13803-13809.

41. Yoshida R, Nagira M, Kitaura M, Imagawa N, Imai T, Yoshie O. Secondary lymphoid-tissue chemokine is a functional ligand for the $\mathrm{CC}$ chemokine receptor CCR7. J Biol Chem. 1998;273:7118-7122.

42. D'Amico G, Frascaroli G, Bianchi G, et al. Uncoupling of inflammatory chemokine receptors by IL-10: generation of functional decoys. Nat Immunol. 2000;1:387-391.

43. Penna G, Sozzani S, Adorini L. Cutting edge: selective usage of chemokine receptors by plasmacytoid dendritic cells. J Immunol. 2001;167:1862-1866.

44. Sanchez-Madrid F, del Pozo MA. Leukocyte polarization in cell migration and immune interactions. EMBO J. 1999;18:501-511.

45. del Pozo MA, Sanchez-Mateos P, Nieto M, Sanchez-Madrid F. Chemokines regulate cellula polarization and adhesion receptor redistribution during lymphocyte interaction with endothelium and extracellular matrix: involvement of CAMP signaling pathway. J Cell Biol. 1995;131:495-508.

46. Zhang Y, McCluskey K, Fujii K, Wahl LM. Differential regulation of monocyte matrix metalloproteinase and TIMP-1 production by TNF-alpha, granulocyte-macrophage CSF, and IL-1 beta through prostaglandin-dependent and -independent mechanisms. J Immunol. 1998;161:3071 3076.

47. Serhan CN, Haeggstrom JZ, Leslie CC. Lipid mediator networks in cell signaling: update and impact of cytokines. FASEB J. 1996;10:1147-1158.

48. Mehindate $\mathrm{K}$, al-Daccak R, Aoudjit $\mathrm{F}$, et al. Interleukin-4, transforming growth factor beta 1 , and dexamethasone inhibit superantigen-induced prostaglandin E2-dependent collagenase gene expression through their action on cyclooxygenase-2 and cytosolic phospholipase A2. Lab Invest. 1996;75:529-538.

49. Thurnher M, Zelle-Rieser C, Ramoner R, Bartsch G, Holtt L. The disabled dendritic cell. FASEB J. 2001;15:1054-1061.

50. Whittaker DS, Bahjat KS, Moldawer LL, ClareSalzler MJ. Autoregulation of human monocytederived dendritic cell maturation and IL-12 production by cyclooxygenase-2-mediated prostanoid production. J Immunol. 2000;165: 4298-4304.

51. Sozzani S, Allavena P, D'Amico G, et al. Differential regulation of chemokine receptors during dendritic cell maturation: a model for their trafficking properties. J Immunol. 1998;161:1083-1086.

52. Thivierge M, Le Gouill C, Tremblay MJ, Stankova J, Rola-Pleszczynski M. Prostaglandin E2 induces resistance to human immunodeficiency virus-1 infection in monocyte-derived macrophages: downregulation of CCR5 expression by cyclic adenosine monophosphate. Blood. 1998; 92:40-45.

53. Zeidler R, Csanady M, Gires O, Lang S, Schmitt B, Wollenberg B. Tumor cell-derived prostaglandin E2 inhibits monocyte function by interfering with CCR5 and Mac-1. FASEB J. 2000;14:661-668.

54. Kalinski P, Smits $\mathrm{HH}$, Schuitemaker $\mathrm{JH}$, et al. IL-4 is a mediator of IL-12p70 induction by human Th2 cells: reversal of polarized Th2 phenotype by dendritic cells. J Immunol. 2000;165: 1877-1881.

55. Katsuyama M, Ikegami R, Karahashi H, Amano F, Sugimoto $\mathrm{Y}$, Ichikawa A. Characterization of the LPS-stimulated expression of EP2 and EP4 prostaglandin $\mathrm{E}$ receptors in mouse macrophage-like cell line, J774.1. Biochem Biophys Res Commun. 1998;251:727-731.

56. Kalinski P, Hilkens CM, Snijders A, Snijdewint FG, Kapsenberg ML. IL-12-deficient dendritic cells, generated in the presence of prostaglandin E2, promote type 2 cytokine production in maturing human naive T helper cells. J Immunol. 1997; 159:28-35. 